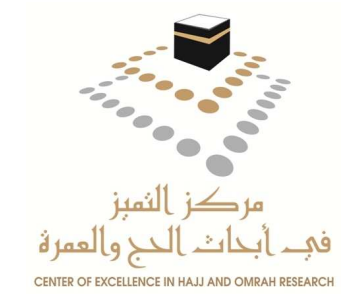

\title{
Intelligent Shelter Allotment for Emergency Evacuation Planning: A Case Study of Makkah
}

Kwangsoo Yang, Faizan Ur Rehman, Hatim Lahza, Saleh Basalamah, Shashi Shekhar, Imtiaz Ahmed, and Arif Ghafoor

October, 2012

\section{Technical Report}

Center of Research Excellence in Hajj and Omrah (Hajjcore)

Umm Al-Qura University

Makkah, Saudi Arabia

Technical Report No. P1104-T1 Oct. 2012 


\section{Intelligent Shelter Allotment for Emergency Evacuation Planning: A Case Study of Makkah}

Kwangsoo Yang ${ }^{1}$, Faizan Ur Rehman ${ }^{2,3}$, Hatim Lahza ${ }^{2,3}$, Saleh Basalamah $^{2,3}$, Shashi Shekhar ${ }^{1}$, Imtiaz Ahmed ${ }^{2,3}$, and Arif Ghafoor ${ }^{4}$

${ }^{1}$ Department of Computer Science and Engineering, University of Minnesota, Minneapolis, USA ksyang,shekhar@cs.umn.edu

${ }^{2}$ College of Computing and Information Systems, Umm Al-Qura University, Makkah, Saudi Arabia fsrehman@uqu.edu.sa, s42704817@st.uqu.edu.sa, smbasalamah,iaahmed@uqu.edu.sa

${ }^{3}$ Center of Research Excellence in Hajj and Omrah (HajjCore), Umm Al-Qura University, Makkah, Saudi Arabia ${ }^{4}$ School of Electrical and Computer Engineering, Purdue University, Indiana, West Lafayette, USA ghafoor@purdue.edu

October 2012 


\begin{abstract}
Given maps of an evacuee population, shelter destinations and a transportation network, the goal of intelligent shelter allotment (ISA) is to assign routes, exits and shelters to evacuees for quick and safe evacuation. ISA is societally important due to emergency planning and response applications in context of hazards such as floods, terrorism, fire, etc. ISA is challenging due to conflicts between movements of evacueegroups heading to different shelters and transportation-network choke-points. State of the practice based on Nearest Exit or Shelter (NES) paradigm addresses the former challenge but not the latter one leading to load-imbalance and slow evacuation. Recent computational development, e.g., capacity-constrained route planning (CCRP), address the latter challenges to speedup evacuation, but do not separate evacuee groups going to different shelter destinations. To address these limitations, we propose a novel approach, namely, Crowd-separated Allocation of Routes, Exits and Shelters (CARES) based on the core idea of spatial anomaly avoidance. Experiments and Hajj case study (Makkah) show that CARES meets both challenges by providing much faster evacuation than NES and much lower evacuee-group movement-conflicts than CCRP.
\end{abstract}

Keywords: evacuate planning, shelter allotment, exit allotment, spatial network database, crowd evacuation, spatial-disjoint allotment

\title{
1 Introduction
}

Given maps of a vulnerable evacuee population, shelter destinations and a transportation network, the goal of intelligent shelter allotment (ISA) is to assign route and destination shelters to evacuee groups that minimize evacuation time under the constraint of spatial disjointness of evacuee groups assigned across different shelters. Spatial disjointness refers to the non-overlapping separation of evacuation zones and is preferred by emergency managers to smooth crowd movement during evacuation and reduce the risk of stampede and compression. ISA is societally important for resilience via emergency planning and response to natural or technical disasters, e.g., floods, hurricanes, tsunami, fire, terrorist acts, industrial accidents, etc. ISA can help emergency planning and response in moving a vulnerable population out of harm's way by recommending allotment of shelters, exits and routes to speed up evacuations while reducing movement-conflict related risks of evacuation slowdowns as well as safety (e.g., compression, stampede, etc).

ISA is challenging due to a variety of reasons including bottlenecks and choke-points in transportation networks (Figure 1(a)), movement-conflicts across evacuee-groups going to different exits or shelters, scale (e.g., large number of evacuees, large size of transportation network) and lack of scientific understanding of crowd movement conflicts.

Early evacuation route planning state of the practice is based on table-top exercises to help emergency managers manually identify and compare alternative allocation of routes, exits and shelters. It often leads to allotments based on a Nearest Exit or Shelter (NES) paradigm [1], which minimizes movement-conflicts across exits and shelters. However, it is difficult for manual processes to identify all choke-points and bottlenecks in transportation networks. Thus, NES risks load-imbalance across routes, exits and shelters leading to unnecessarily high evacuation times as shown in Figure 1(b).

Computational approaches for evacuation route planning may be categorized into Microscopic Simulation [2, 3] Mathematical Programming [1, 4], and Capacity-Constrained Route 


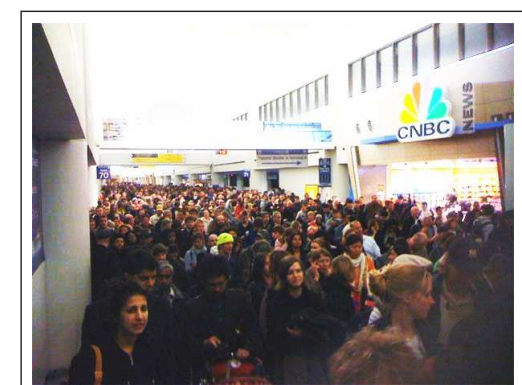

(a) Newark Airport evacuation (Courtesy:

http://www.doobybrain.com)

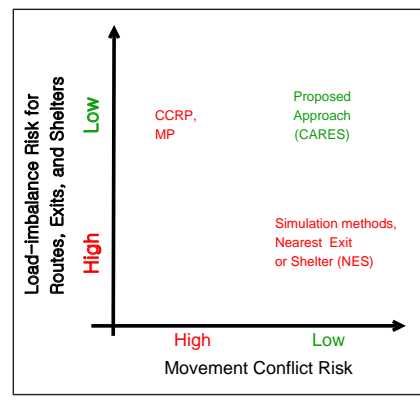

(b) Comparison of goals ( of proposed approach with the city of Makkah, Saudi Arabia those of related work

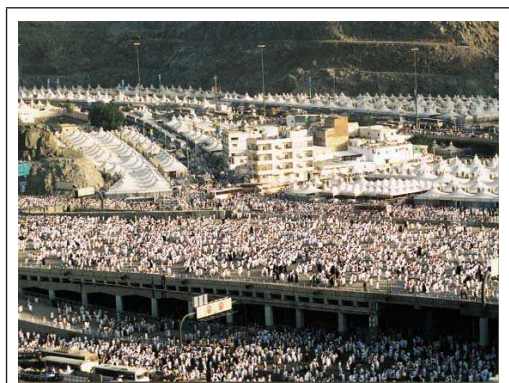

(Courtesy:

http://www.saudiembassy.ne)

Figure 1: Nearest shelter may violate capacity constraint of shelters (Best in color)

Planning [5, 6, 7]. Micro-scopic Simulation methods [2, 3] model behavior of individuals (e.g., drivers or pedestrians) using agent-based models under the assumption of perfect information and repeated experience (e.g., commute) to derive game-theoretic Wardrop's equilibrium [8]. Such approaches are computationally exorbitant even for medium-size evacuation scenarios with tens of thousands of evacuees. In addition, their assumption of repeated experience leading to Wardrop equilibrium and perfect information does not hold for rare events such as emergency evacuations.

To reduce computational costs, Mathematical Programming (MP) methods $[1,4]$ simplify the models by examining macro scopic traffic-flow behavior along transportation flow networks and ignoring movement conflicts at route intersections. They define a mathematical program (e.g., linear, integer or quadratic program) on a time-expanded graph, which replicates the transportation flow-network model for every time-unit during evacuation. Unfortunately, the computational cost are too high for use with large evacuation scenarios with hundreds of thousands (or millions) of evacuees. In addition, traditional MP approaches require the user to set an upper limit on evacuation time in order to derive a solution, which may be difficult for end-users.

Capacity-Constrained Route Planning (CCRP) [5, 6, 7] addresses some of these limitations through the use of time-aggregated graphs to dramatically reduce storage needs of time-expanded graphs. This state of art approach leverages spatio-temporal shortest path algorithms and reservations instead of mathematical programming to further reduce computational costs. Experimental evaluations and field-tests show that CCRP is computationally orders of magnitude faster than Mathematical Programming, while yielding comparable solution quality. However, CCRP does not model movement conflicts across evacuee groups going to different shelters. CCRP solutions may include criss-crossing routes for evacuee groups heading to different exits or shelters. Thus, CCRP has high movement conflict across routes, exits and shelters as summarized in Figure 1(b).

This article proposes a novel approach, namely, Crowd-separated allotment of Routes, Exits and shelters (CARES), based on the core ideas of detection and avoidance of spatial anomalies. Spatial anomalies are evacuation-units (e.g., a evacuee group) whose allotted route, exit or shelter is different than those of the vast majority of its spatial neighbors. Assuming that spatial anomalies are indicators of potential movement-conflicts, CARES identifies them and re-allots their route, exit, and shelter. Experimental evaluation and a 
Hajj case study in the city of Makkha (Saudi Arabia) show that CARES leads to significantly lower movement conflicts relative to CCRP and much faster evacuation than NES.

Scope: This article primarily addresses the challenges of computational scalability, transportation network choke-points and movement conflict in the context of the intelligent shelter allocation problem. The research informally leverages a century's worth of crowd control experience at Makkah (Figure 1(c)). However, formal consideration of crowd movement physics is beyond the scope of the present study. Other considerations outside the scope include realtime situation assessment to update maps of evacuees and transportation networks, as well as modeling of evacuee behavior (e.g., panic), disaster physics (e.g., flooding of routes), and dependency of flow-rate on evacuee density.

Outline: The rest of this article is organized as follows. The ISA problem is formally defined in Section 2 and the proposed CARES algorithm is described in Section 3. Section 4 presents results of experimental evaluation of CARES and Section 5 reports a case study. Finally Section 6 presents conclusions and future work.

\section{Problem Statement}

In our problem formulation, a transportation network is represented and analyzed as a graph composed of nodes and edges. Every node represents a spatial location in geographic space with a time-dependent node capacity. Every edge represents a connection between two nodes and has a travel time with a time-dependent edge capacity. Source nodes have a number of evacuees and shelter (destination) nodes have a capacity for evacuees. The Intelligent Shelter Allotment (ISA) problem can be formalized as follows:

Given: A transportation network with non-negative integer capacity constraints on nodes $N$ and edges $E$, the total number of evacuation units and their locations, and locations and capacity of all evacuation shelters (destinations).

Output: An evacuation plan consisting of a set of origin-destination routes and a spatialdisjoint allotment of sources across destinations.

Objective: (1) Minimize the computational cost of producing the evacuation plan. Minimize the evacuation time.

Constraints: (1) Source zones are allocated across different shelters (Spatially disjoint). (2) The scheduling of evacuees on each route observes the capacity constraints. (3) Edge travel time preserves FIFO (First-In First-Out) property. (4) Computer memory is limited.

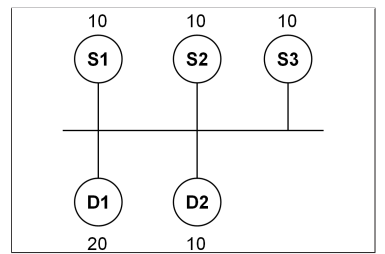

(a) Input

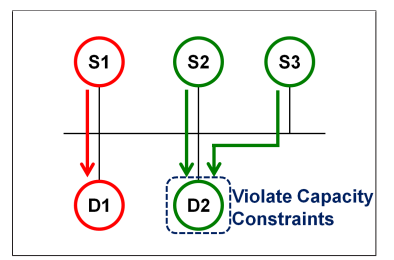

(b) Allotment 1 (Invalid)

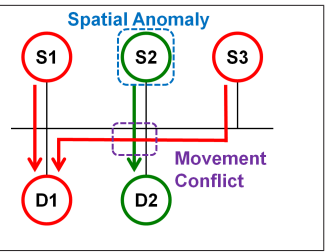

(c) Allotment 2

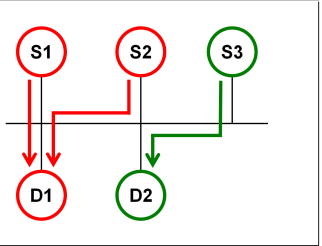

(d) Allotment 3

Figure 2: Example of ISA input and possible outputs (Best in color)

Figure 2 illustrates input and outputs of ISA with a transportation network with three source nodes $(S 1-S 3)$ each with 10 evacuees and two shelter destinations $(D 1-D 2)$ with shelter capacities of 20 and 10 respectively. Figure 2(b) shows an invalid allotment mimicing nearest exit and shelter (NES) paradigm. Even though this method avoids movement 
conflicts, it does not honor the capacity constraint of shelter D2 and may lead to congestion at $D 2$.

Figure 2(c) shows a valid allotment honoring shelter capacity constraints. Every source node except $S 3$ is allotted their nearest shelter. This allotment, however, exhibits crisscrossing of routes for sources $S 2$ and $S 3$ leading to potential movement conflicts. In addition, shelter destination $D 2$ allotted to source $S 2$ is different from the shelter destination $D 1$ allotted to its neighboring sources $S 1$ and $S 3$. Thus, source $S 2$ is considered a spatial anomaly.

Figure 2(d) shows another valid allotment honoring shelter capacity constraints. It also reduces movement conflicts and eliminates spatial anomalies. In the next section, we propose an algorithm to find such shelter allocations.

\section{Proposed Algorithm}

The Crowd-separated Allotment of Routes, Exits and Shelters (CARES) algorithm is based on the core ideas of spatial neighborhoods, spatial anomaly avoidance, and source node priority. Spatial neighborhood is a relationship defined on the set of source nodes. A source node $S 1$ may be considered a neighbor of $S 2$ if it is within a given geographic- or roaddistance. Alternative definitions may be based on $K$-nearest neighbors given an integer $K$.

Spatial anomaly is an indicator of movement conflicts using local comparison of a source node with neighboring source nodes. The comparison may be based on allotted exits, routes or shelters. A source is considered a spatial anomaly if its allotted exit, shelter or route is very different from those of the majority of its neighbors. For simplicity, we use a comparison based on allotted shelters. Recall that source $S 2$ in Figure 2(c) is considered a spatial anomaly as its allotted shelter is different from the shelter allotted to its neighboring sources.

Source node priority may be based on urgency (e.g., distance to an incident triggering the evacuation), ease of evacuations (e.g., distance to nearest shelter), ease of avoiding spatial anomalies (e.g., number of neighbors with allotted shelters), etc.

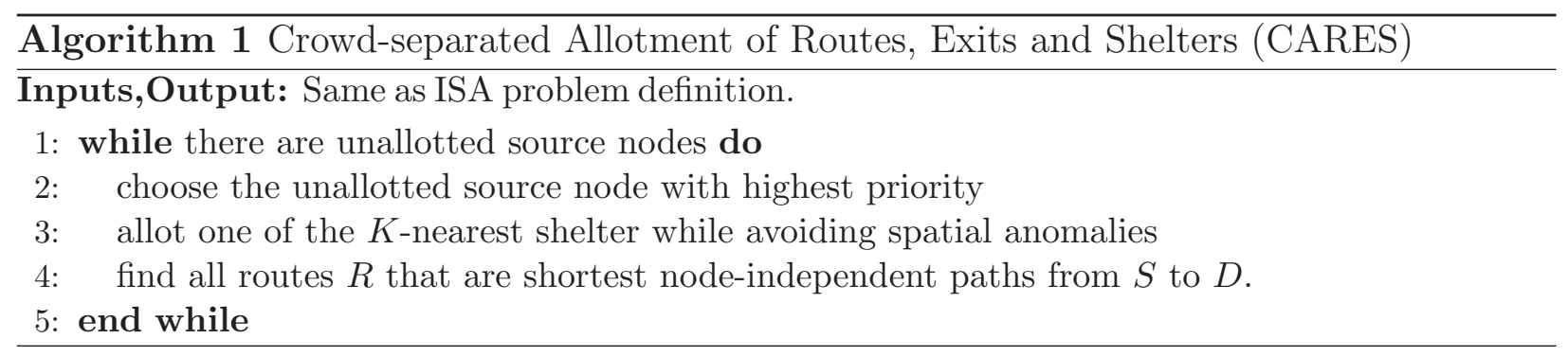

Algorithm 1 shows an abstract description of the CARES approach. In each iteration, the algorithm first identifies the highest priority source node with no current allotment. It then determines the appropriate allotment of a route, an exit and a shelter for the selected source while avoiding spatial anomalies. This may be accomplished by examining the intersection of the set of $K$-nearest shelters for the selected node with the set of shelters allocated to neighboring source nodes. If there is a potential spatial anomaly then the allotment for the selected node and its neighbors is reconsidered to remove the spatial anomaly.

Let us trace the execution of CARES with the ISA problem instance shown in Figure 2. For simplicity, we use a source priority based on ease of evacuations (e.g., distance to nearest shelter). Ties may be broken based on the lexical order of source node identifiers. In the 
first iteration, CARES may choose source node $S 1$ and assign it the nearest shelter $D 1$, updating the remaining capacity of $D 1$ to 10 . In the second iteration, CARES may choose source node $S 2$, initially allotting it to the nearest shelter $D 2$.

In the third iteration, CARES may choose source node $S 3$ and consider assigning it to the only available shelter $D 1$. However, this may lead to spatial anomaly $S 2$, triggering a revision of allotments for $S 3$ and its neighbor $S 2$. Given that re-allotment of shelter for $S 3$ is not feasible without re-allotment for $S 2$, CARES will re-allot $S 2$ to shelter destination $D 1$. Consequently, $S 3$ is allotted shelter destination $D 2$, leading to the allotment shown in Figure 2(d).

CARES's computational cost and its ability to reduce movement conflicts is sensitive to the integer $K$ used to define the neighborhood for identifying and avoiding spatial anomalies. A smaller value of $K$ reduces computational cost as well as the ability to avoid movement risk. The special case of CARES for $K=0$ behaves like the Capacity-Constrained Route Planning algorithm. Larger values of $K$ increase computational cost but reduce the risk of movement conflicts.

\section{Experimental Evaluation}

The main goal of our experiments was to compare the proposed CARES algorithm with stateof-the-art CCRP algorithms and state-of-the-practice NES paradigm towards meeting the ISA challenges of managing transportation choke-points and avoiding movement conflicts. A secondary goal was to evaluate the scalability of the proposed algorithms for large evacuation scenarios with hundreds of thousands of evacuees and varying crowd density.

Comparison Metrics: We used three metrics to measure of an algorithm's ability to manage transportation choke-points: (a) evacuation time, i.e., the time for the last evacuee to reach their allotted shelter, (b) shelter arrival rate over the duration of evacuation, and (c) cumulative precentage of population reaching shelter. For the case study (described in the next section), we use visual maps to identify spatial anomalies, e.g., evacuation groups whose allotted shelters differ from those of its neighbors, to compare algorithms ability to avoid movement conflicts.

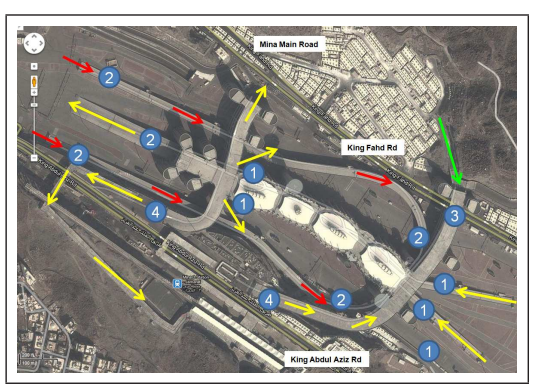

(a) Jamarat complex has multiple (b) Crowd density and walking

levels shown as 1, 2, 3 and 4 on speed model of Hajj pilgrims [9] associated ramps. Every level is a distinct shelter destination. (Courtesy: https://maps.google.com/)

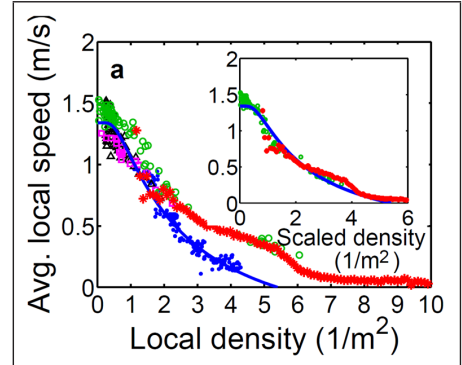

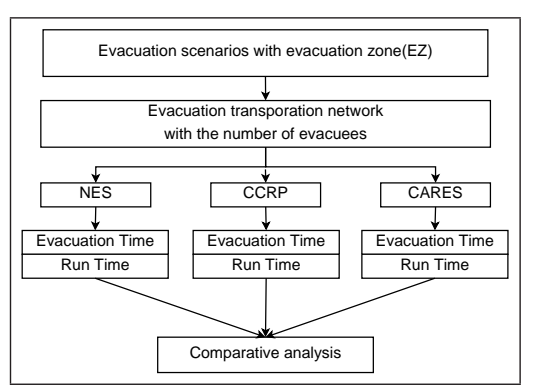

(c) Experiment design

Figure 3: Experiment setup for evaluating the proposed CARES algorithm (Best in color)

Benchmark: Our experiments used a flash-flood scenario around the Jamarat complex in 
the tent city of Minna for Hajj in Makkah, Saudi Arabia. Overall, the Minna tent-city was modeled using 15,578 nodes representing tents and 38,490 edges representing walkways, roads, ramps, etc. using OpenStreetMap ${ }^{1}$. The area affected by the flash-flood was defined around a subset of the tent-city at the foothills near the Jamarat complex include 160 tent groups with 624 individual tents. These tent groups are linked by walkways around $2 m$ wide. These walkways are connected by walkways, ramps, roads (9- $m$ wide road) and highways (e.g., 11-m wide King Fahd Road) to Jamarat complex. The destination shelters were defined by two higher levels (levels 2 and 3) of the Jamarat complex structure as shown in Figure 3(a) using red and green arrows.

Experiment Design: Variable parameters included number of evacuees (50, 000 to 250, 000 in increments of 50,000), and crowd density (1 to 4 people $\left./ \mathrm{m}^{2}\right)$. The walking speed was determined from crowd density using previous studies of Makkah crowd movement (summarized in Figure 3(b)), and the slopes of ramps and escalators. Fixed parameters included $K$, the number of neighbors needed to determine spatial anomalies. Its value was fixed at 20 for CARES algorithm for all experiments. The NES, CCRP and CARES algorithms were implemented in Java 1.7 with 1 GB memory run-time environment. All experiments were performed on an Intel Core i7-2670QM CPU machine running MS Windows 7 with 8 GB of RAM. Figure 3 summarizes the overall experimental design.

Experimental Results: Figure 4 reports experimental results illustrate effects of number of evacuees and crowd density on computational cost (i.e., run-time) and three metrics of an algorithm's ability to manage transportation choke-points: (a) evacuation time, (b) shelter arrival rate over the duration of evacuation, (c) cumulative percentage of population reaching shelter.

Effect of the number of evacuees on evacuation time and computational cost: Figure 4(a) and Figure 4(c) show the effect of number of evacuees on evacuation time and computational cost of NES, CCRP and CARES. Crowd density was fixed at (1 people $/ \mathrm{m}^{2}$ ) leading to a walking speed of $1 \mathrm{~m} / \mathrm{sec}$ [9]. For all algorithms, evacuation time increases with growth in evacuee population. Overall, CARES leads to much lower evacuation times but higher computational costs relative to NES. CARES has a slightly longer evacuation time and higher computational cost than CCRP due to its focus on avoiding movement conflicts. Effect of crowd density on evacuation time and computational cost: Figure 4(b) and Figure 4(d) show the effect of crowd density (and associated walking speed) on evacuation time and computational cost of NES, CCRP and CARES. We fixed the number of evacuees $(150,000)$ and varied the crowd density from 1 to 4 people $/ \mathrm{m}^{2}$. Associated walking speed varied from $1 \mathrm{~m} / \mathrm{sec}$ down to $0.45 \mathrm{~m} / \mathrm{sec}$ [9]. For all algorithms, evacuation time increases as crowd density grows, Trends are similar to previous experiment with CARES having much lower evacuation times but higher computational costs than NES. CARES has a slightly higher evacuation time and higher computational cost than CCRP due to its focus on avoiding movement conflicts.

Comparison of Shelter Arrival Rates and Cumulative Percentage: Figure 4(e) and Figure 4(f) respectively show the cumulative percentage reaching shelter and shelter arrival rates for of NES, CCRP and CARES. We fixed the number of evacuees to 150,000 and crowd density to 1 people $/ \mathrm{m}^{2}$. We assigned the capacities of each shelter entrance to 600 people/min (level 2) and 360 people/min (level 3). Overall, CCRP and CARES have similar performance and outperform NES, possibly because NES does not consider capacity constraints on routes and shelters. All algorithms show three phases of evacuation, pre-

\footnotetext{
${ }^{1}$ http://www.openstreetmap.org/, Retrieved Oct. 2012
} 
steady-state, steady-state and post-steady-state. The steady-state shows up as a flat shelter arrival rate in Figure 4(f) and as a linear rate of shelter arrival in Figure 4(e). The highest shelter arrival rate of both CCRP and CARES is 960 people/min because they use both shelters (level 2 and level 3). We can see that all three algorithms saturated the capacity of shelter entrances.

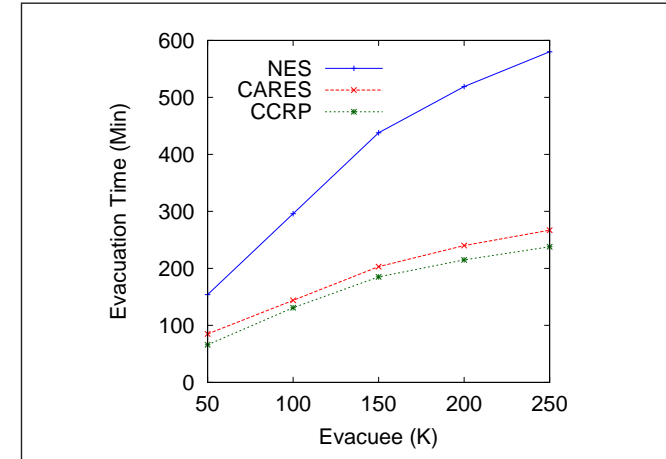

(a) Effect of the number of evacuees (1 people $/ m^{2}$ )

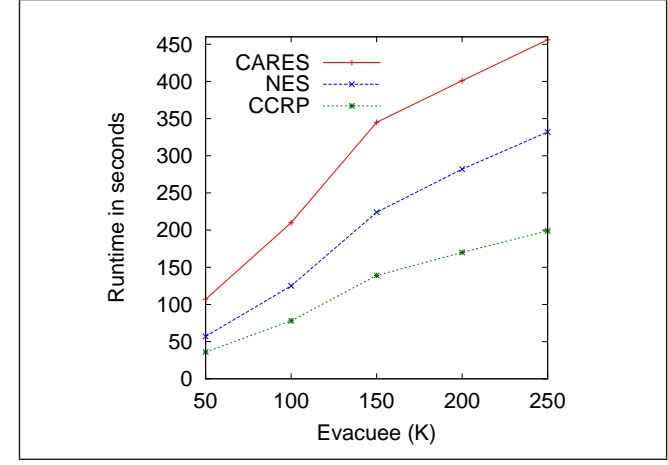

(c) Runtime comparison (1 people $/ \mathrm{m}^{2}$ )

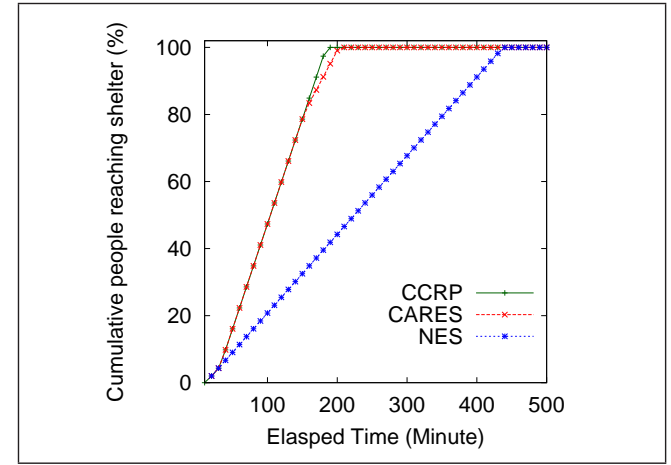

(e) Cumulative evacuees (\%) (150,000 evac- (f) uees, 1 people $/ \mathrm{m}^{2}$ )

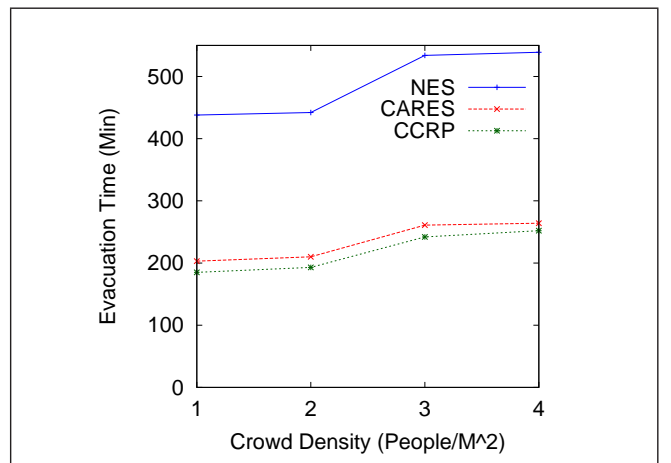

(b) Effect of crowd density (150,000 evacuees)

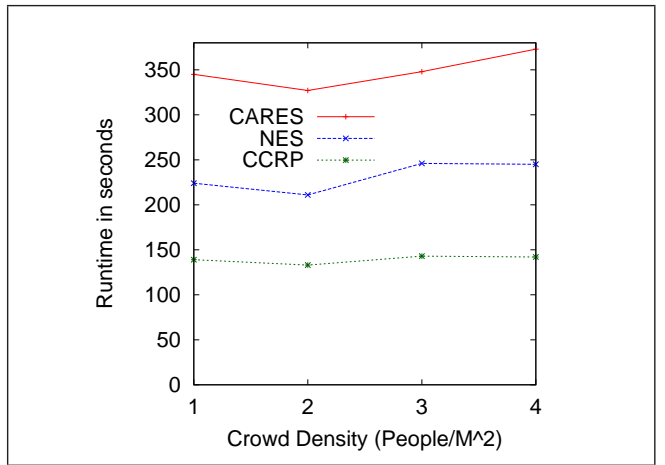

(d) Runtime comparison (150,000 evacuees)

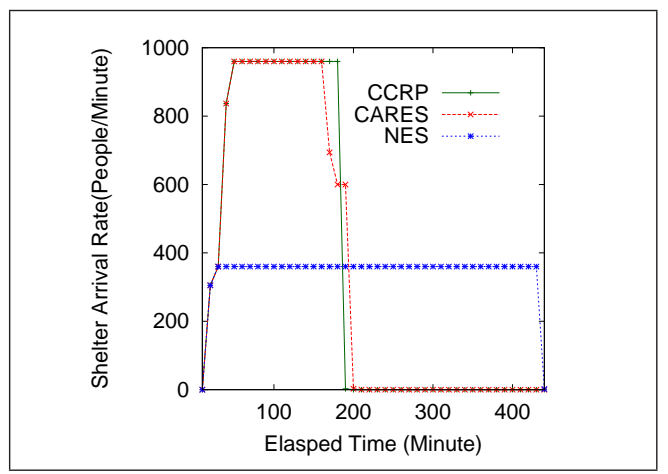

(f) Shelter arrival rate (people/minute) (150, 000 evacuees, 1 people $\left./ \mathrm{m}^{2}\right)$

Figure 4: Experimental Results (Best in color)

\section{Case Study with A Hajj Scenario}

In this section, we report on a case study of CARES for Intelligent Shelter Allotment. The scenario is a flash-flood that affects a small part of Mina tent city during the Hajj pilgrimage. 
Hajj and Mina Tent City near Makkah, Saudi Arabia: It has been a daunting challenge for the Kingdom of Saudi Arabia to manage around three million Hajj pilgrims of multi-nationalities with multi-cultural and multi-linguist disposition, especially under the recent multimedia environment. Many times lack of proper enforcement lead to disastrous situations resulting in numerous causalities, which were neither properly recorded nor accurately reported. However, major incidents have been covered by media and summary of its reporting are also available on the Wikipedia entry on Hajj ${ }^{2}$.

In addition, Mina has experienced a variety of fatal disaster scenarios in the past. The exponential growth in pilgrims and global environmental changes have resulted in recurring record breaking events that further enhance potential vulnerability to a wide variety of disasters, including, floods, fire, stampede, terrorists activities, epidemics, collapse of structure/ramp, rock falls, etc. Of these, historically recurring floods have been widespread and highly disruptive, even life threatening. Being surrounded by high mountains remains highly vulnerable to floods due to short duration high intensity rainfall. The northern part is more threatened being close to higher mountains and somewhat bigger catchment areas, and the tents closer to mountains will be exposed to flash floods (kind of hill torrents) with rock falls/stone debris, generally of short duration but very violent. Areas in the valleys, on the other hand, could be more disrupted but less dangerous, especially since the drainage of Mina has been improved.

A possible shelter to protect pilgrims from flash-flood is the Jamarat complex. The complex is a ground plus 4-level structure, served by ramps connecting each level with oneway flow, a great improvement over the past practices. Movement toward Azizyah/Haram is allowed, however cross movements are restricted. Metro station located next to Jamarat is connected to 4th level. Various levels are also linked with stairs and electric escalators for any emergency movements. Out of four days at Mina, Jamarat can be used for relief activities one day only (i.e, 8th Dhul-Hijjah) as a staging place for victims, whereas, for remaining three days (i.e, 10-12 Dhul-Hijjah) it will be heavily crowded and can only serve as a transit point for evacuation by Metro/bus/helicopter/on foot to Azizyah/Haram.

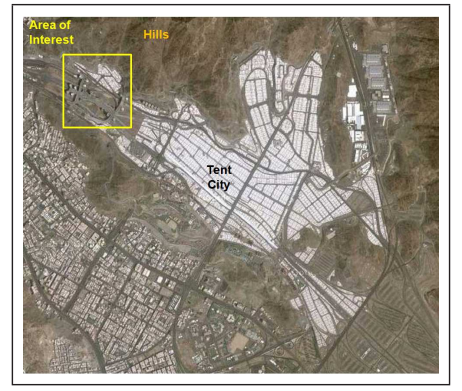

(a) Area of interest within Tent city

(Courtesy: Google Maps)

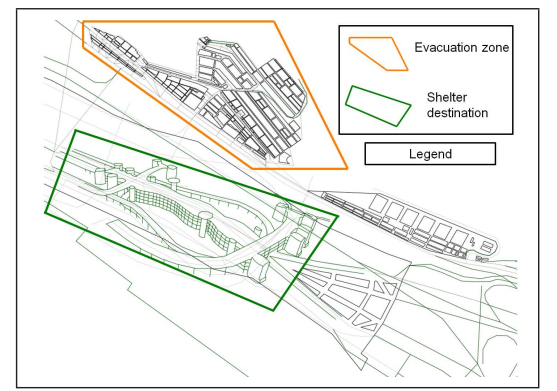

(b) Evacuation zone and shelter destination (Jamarat complex) in area of interest

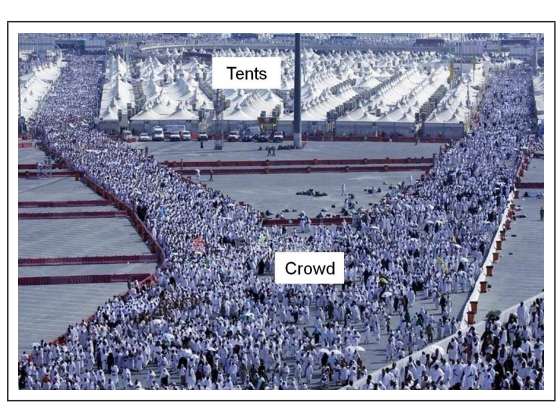

(c) crowd walking from tent city to Jamarat complex

(Courtesy: http://www.flickr.com/)

Figure 5: Jamarat complex and Surrounding Area in Mina (Best in color)

Flash Flood Scenario: Mina is a rough mountain area situated to the south-east of Makkah. It is a narrow valley about six kilometers from the Sacred Mosque of Makkah on the way to Arafat. It covers approximately 812 hectares within the holy boundaries, $52 \%$ of which is flat land. The pilgrims have to stay here for at least four days and three nights as

\footnotetext{
${ }^{2}$ http://en.wikipedia.org/wiki/Incidents_during_the_Hajj
} 
part of Hajj rituals. All of Mina is covered with Teflon coated tents to provide temporary accommodation to the pilgrims, thus termed as the world's biggest tent city. There are around 6,000 tents in approximately $20 \mathrm{~km}$-covered area of Mina. In our analysis, we chose 160 tent groups in approximately $1 \mathrm{~km}$-covered area. Then we assigned 50, 000 evacuees for this area and used 4 people $/ \mathrm{m}^{2}$ crowd density.

Figure 5(a) shows all Mina tents and our area of interest for the flash flood scenario. Figure 5(b) shows evacuation zone for the tent city (orange color) and the shelter destination of Jamarat complex (green color). Recall that Jamarat complex is a ground plus 4-level structure, served by ramps or escalators connecting each level with one-way flow. Evacuees from the tent city use either ramps (Figure 5(c)) or escalators to enter into the complex.

Movement-conflict Comparison of Allotment Algorithms: Figure 5 shows the shelter allotments provided by NES, CCRP and CARES for two colors, namely, red for shelter at level 2 and green for shelter on level 3 of Jamarat complex. Tents and routes are colored by the shelter destination. Overall, CCRP allotment exhibits spatial anomalies as highlighted in Figure 6(b) via red circle indicating movement conflicts. In contrast, NES and CARES shelter allotments show no spatial anomalies indicating ability to avoid movement conflict. Figure 6(a) shows that NES uses only one shelter (level 3) due to its proximity to all source nodes. This may explain the longer evacuation time for NES allotments in the experimental results presented in previous section.

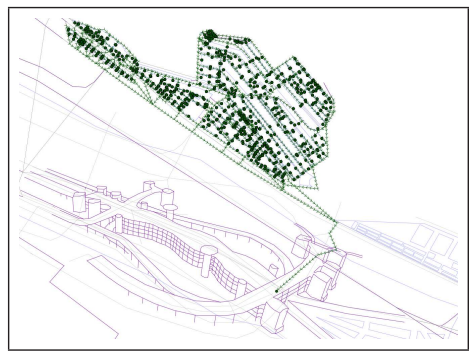

(a) NES Allotment

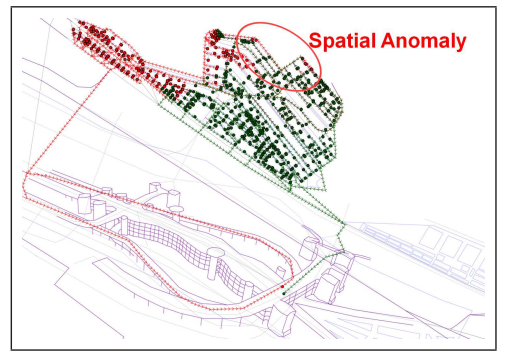

(b) CCRP Allotment

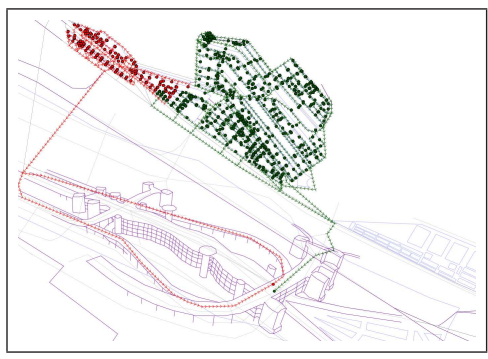

(c) CARES Allotment

Figure 6: Shelter allotments (and routes) recommended by three algorithms $(50,000$ evacuees, 4 people $/ m^{2}$ ) (Best in color)

Visualizing Spatial Distribution of Shelter Arrival Time: Figure 7 shows the spatial distribution of shelter arrival time for NES, CCRP and CARES. Map visualization may be useful in identifying areas which are hard to evacuate and which could benefit most from the addition of new routes to shelters. All three allotments show that the area highlighted by red circles near top center has highest values for shelter arrival time, despite its proximity to foothills and flash-floods. If a shelter's arrival time is unacceptable, authorities may consider adding additional routes to speed up evacuations from this area. Figure 7(a) also shows that NES allots many tents with very high evacuation time relative to CCRP (Figure 7(b)) and CARES (Figure 7(c)).

\section{Conclusions and future work}

Intelligent Shelter Allotment (ISA) for evacuation route planning is important for dealing with natural and technological disasters. ISA is challenging due to conflicting requirements of minimization of evacuation time while avoiding movement conflicts. In this paper, we 


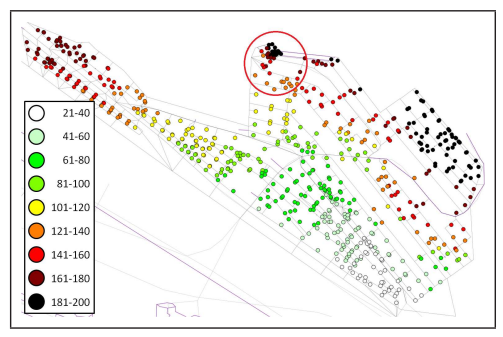

(a) NES Allotment

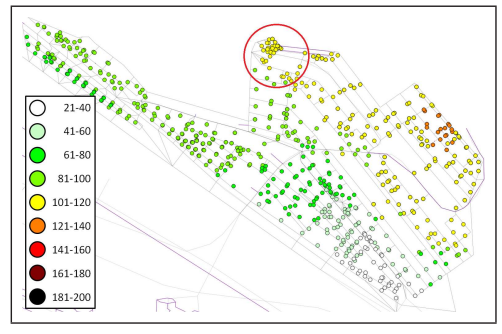

(b) CCRP Allotment

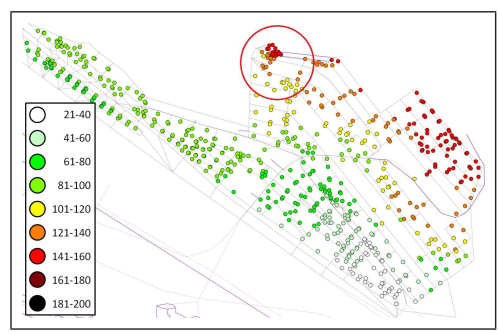

(c) CARES Allotment

Figure 7: Distribution of shelter arrival times for evacuee groups for three algorithms (50,000 evacuees, 4 people $/ \mathrm{m}^{2}$, evacuation unit $=$ tent, circle color $=$ evacuation time $)$

(Best in color)

introduced a novel approach, known as Crowd-separated Allotment of Routes, Exits, and Shelters (CARES), which intelligently minimized evacuation time under constraint of spatial disjointness of evacuee groups assigned across different shelters. Experimental and case study based evaluation shows that CARES meets the dual challenges.

In future work, we would like to expand experiments to study the effect of additional parameters such as $K$, the number of neighbors. We will also analyze the computational complexity of ISA and CARES, identify computational bottlenecks, and explore alternative design decisions to speed up CARES. In addition, we plan to explore direct indicators of movement conflicts such as route overlap and design global methods to avoid such conflicts.

\section{Acknowledgments}

The research presented in this paper has been supported by the Center of Research Excellence in Hajj and Omrah (HajjCore), Umm Al-Qura University, Makkah, Saudi Arabia under project number P1104, entitled (A Real-time Route Planning and Emergency Evacuation Management Tool for Hajj \& Umrah Activities), the US National Science Foundation, and USDOD. We also extend thanks to the University of Minnesota Spatial Databases and Spatial Data Mining Research Group for numerous suggestions to improve technical quality of the paper. We thank Kim Koffolt for improving the readability of this paper.

\section{References}

[1] H. Hamacher and S. Tjandra, "Mathematical modelling of evacuation problems-a state of the art," Pedestrian and Evacuation Dynamics, vol. 2002, pp. 227-266, 2002.

[2] M. Ben-Akiva et al., "Development of a deployable real-time dynamic traffic assignment system: Dynamit and dynamit-p users guide," Intelligent Transportation Systems Program, Massachusetts Institute of Technology, 2002.

[3] H. Mahmassani, H. Sbayti, and X. Zhou, "Dynasmart-p: Intelligent transportation network planning tool: Version 1.0 users guide," College Park, MD: Maryland Transportation Initiative, University of Maryland, 2004. 
[4] L. Fleischer and M. Skutella, "Quickest flows over time. SIAM Journal on Computing," vol. 36, no. 6. [Philadelphia] Society for Industrial and Applied Mathematics., 2007, pp. $1600-1630$.

[5] X. Zhou, B. George, S. Kim, J. Wolff, Q. Lu, S. Shekhar, O. Nashua, and G. Team, "Evacuation planning: A spatial network database approach. bulletin of the technical committee on data engineering, vol.33 no.2." IEEE Computer Society, 2010, p. 26.

[6] Q. Lu, B. George, and S. Shekhar, "Evacuation route planning: a case study in semantic computing." Int. J. Semantic Computing, vol. 1, no. 2, pp. 249-303, 2007.

[7] S. Kim, S. Shekhar, and M. Min, "Contraflow transportation network reconfiguration for evacuation route planning," Knowledge and Data Engineering, IEEE Transactions on, vol. 20, no. 8, pp. 1115-1129, 2008.

[8] J. Wardrop, "Some theoretical aspects of road traffic research," in Proceedings of the Institution of Civil Engineers, 2(1). Thomas Telford Ltd., 1952.

[9] D. Helbing, A. Johansson, and H. Al-Abideen, "Dynamics of crowd disasters: an empirical study," Physical review E, vol. 75, no. 4, p. 046109, 2007. 


\section{A Proof of NP-Completeness}

In this section, we prove the NP-completeness of the Intelligent Shelter Allotment (ISA) problem.

Lemma 1. ISA problem is NP-complete

Proof. Let $\operatorname{dest}(s)$ be a destination of evacuation routes from source $s$, let zone $(s)$ be a zone containing source $s$, and let $s z(s)$ be a set of source nodes that zone $(s)$ contains. To show that ISA $\in \mathrm{NP}$, for a given spatio-temporal network $S T N=(V, E, T)$, a pair of $\left(s_{i}, t_{i}\right)$, the number of unit of evacuation $u_{i}$, capacity constraints $c(e \in E)$, we use the set of feasible flows $f\left(s_{i}, t_{i}\right)$ as a certificate for $S T$. We can check whether $f\left(s_{i}, t_{i}\right) \geq u_{i}$ with evacuation time $t$ and $s z\left(s_{i}\right)$ have a same destination $\operatorname{dest}\left(s_{i}\right)=t_{i}$ in polynomial time. Obviously, the ISA $\in$ NP since a nondeterministic algorithm produces all possible flows $f\left(s_{i}, t_{i}\right)$ and checks in polynomial time that $f\left(s_{i}, t_{i}\right) \geq u_{i}$ and a spatial-disjoint allotment of sources across destinations.

The edge-disjoint paths (EDP) problem is defined as: Given a graph $G=(V, E)$ and terminals $s_{1}, t_{1}, s_{2}, t_{2}, \ldots s_{k}, t_{k} \in V$, the goal is to find disjoint paths between $s_{i}$ and $t_{i}$. The directed edge-disjoint paths problem is NP-complete ${ }^{3}$.

We transform EDP to ISA. The reduction algorithm begins with an instance of ISA. Suppose that every source zone contains only one source and the capacity $c(e \in E)$ is 1 . We can easily build this graph in polynomial time. We claim that given $S T N=(V, E, T)$, the feasible flows $f\left(s_{i}, t_{i}\right) \geq u_{i}$ within a evacuation time $t$ is EDP.

\footnotetext{
${ }^{3}$ J. Vygen, "Np-completeness of some edge-disjoint paths problems," Discrete Applied Mathematics, vol. 61, no. 1, pp. 83-90, 1995.
} 


\section{B Problem Statement}

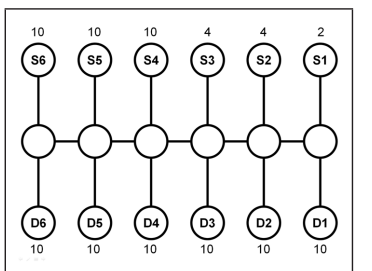

(a) Input

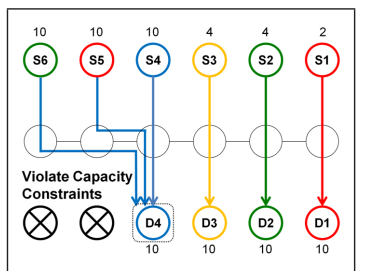

(b) Allotment 1 (Invalid)

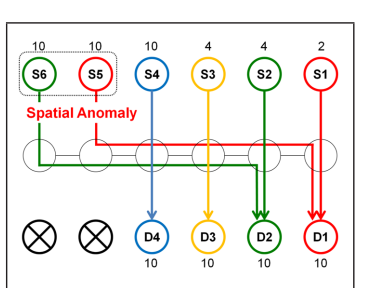

(c) Allotment 2

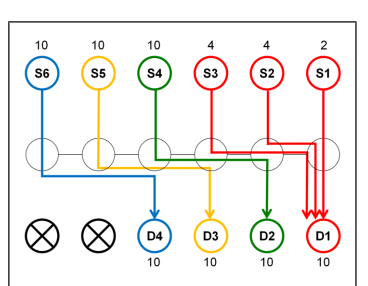

(d) Allotment 3

Figure 8: Example of ISA input and possible outputs (Best in color) 


\section{Experimental Results}

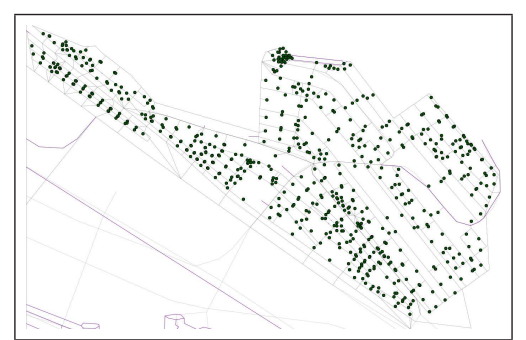

(a) NES

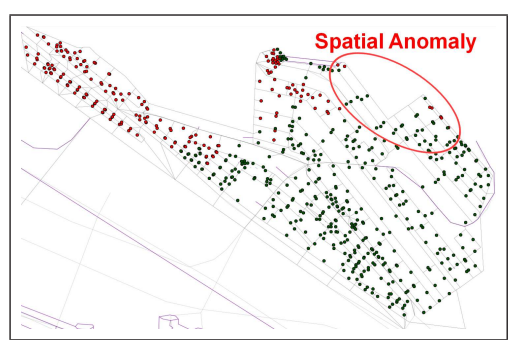

(b) CCRP

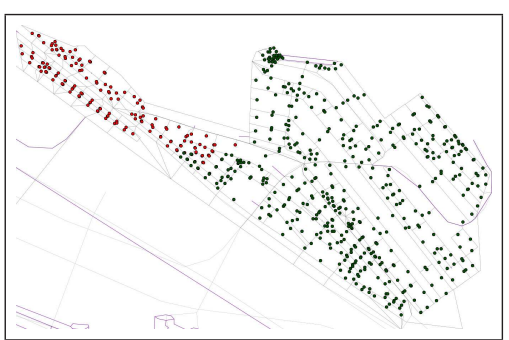

(c) CARES

Figure 9: Shelter Allotment Comparison (50,000 evacuees, 4 people $/ \mathrm{m}^{2}$ )(Best in color) 


\section{Disasters at Mina in Historic Perspective}

It has been a daunting challenge for the Kingdom to manage around three million pilgrims of multi-nationalities with multi-cultural and multi-linguist disposition, especially under recent multimedia environment. Many times lack of proper enforcement lead to disastrous situations resulting in numerous causalities, which were neither properly recorded nor accurately reported. However, major incidents have been covered by media and summary of its reporting are also available on the Internet ${ }^{4}$.

Incidents of Stampedes: The details reported by media included:

- July 2, 1990: a stampede inside in a pedestrian tunnel (namely Al-Ma'aisim) leading out from Mecca towards Mina and Arafat led to huge casualties.

- May 23, 1994: a stampede killed at least 270 pilgrims at the stoning of the Devil ritual.

- April 9, 1998: at least 118 pilgrims were trampled to death and 180 injured in an incident on Jamarat Bridge.

- March 5, 2001: thirty-five pilgrims were trampled to death in a stampede during the stoning of the Devil ritual.

- February 11, 2003: The stoning of the Devil ritual claimed lives of 14 pilgrims.

- February 1, 2004: 251 pilgrims were killed and another 244 injured in a stampede during the stoning ritual in Mina.

- March 5, 2001: thirty-five pilgrims were trampled to death in a stampede during the stoning of the Devil ritual.

- January 12, 2006: A stampede during the ritual Ramy at Jamar?t on the last day of the Hajj in Mina killed at least 346 pilgrims and injured at least 289 more shortly after 13:00 pm local time, an estimated two million people were performing the ritual at the time.

Incidents of Fires: It reported in the media that following incidents occurred when millions of pilgrims were gathered for performing Hajj rituals at Mina involving exposion of gas cylinder, electricity wiring, etc:

- December 1975: An exploding gas cylinder caused fire in a tent colony, resulting in the deaths of 200 pilgrims.

- April 15, 1997: 343 pilgrims were killed and 1,500 injured in a tent fire at Mina on 8 Dhu'l-Hijja between 10 am to 12 pm, though tents are now fireproof.

- November 1, 2011: two pilgrims died in a coach fire, whereas the remaining got out and the victims were still struggling while the coach exploded three times.

Protests and Violence: The following incidents of protest, violence and external terrorism have been can also occur by the external bodies like terrorism.

\footnotetext{
${ }^{4}$ http://en.wikipedia.org/wiki/Incidents_during_the_Hajj
} 
- November 20, 1979: A group of about 200-500 militants occupied the Grand Mosque, and later were expelled leaving about 250 dead and 600 wounded.

- July 31, 1987: Iranian pilgrims rioted, causing the deaths of over 400 people.

- July 9, 1989: Two bombs exploded, killing 1 pilgrim and wounding another 16.

Potential Disaster Vulnerabilities: Sequel to above, it is evident that Mina has experienced a variety of fatal disaster scenarios in the past. Whereas, exponential growth in pilgrims and global environmental changes resulting in recurring record breaking events would further enhance the potential vulnerability to a wide variety of disasters, including, floods, fire, stampede, terrorists activities, epidemics, collapse of structure/ramp, rock falls, etc. Off these, historically recurring floods have been widespread and highly disruptive, even life threatening. Mina being surrounded by high mountains remains highly vulnerable to floods consequent to short duration high intensity rainfalls, northern part more threatened being close to higher mountains and somewhat bigger catchment areas, especially the tents closer to mountains will be exposed to flash floods (kind of hill torrents) with rock falls/stone debris, generally of short duration but very voilent. Whereas, areas in the valleys could be more disrupted but less dangerous, especially since the drainage of Mina has been improved. However, it would be difficult to undertake precise risk assessment consequent due to absence of historic data on precipitation/runoff and lack of accurate surveys/digital elevation model of the area. 


\section{E Experimental Parameters - Mina}

Mina is a rough mountain area situated to the south-east of Makkah. It is a narrow valley about six kilometers from the Sacred Mosque of Makkah on the way to Arafat. It covers approximately 812 hectares within the holy boundaries, $52 \%$ of which is flat land. The pilgrims have to stay here for atleast 4 days and three nights as part of Hajj rituals, i.e, on 8th, 10th, 11th and 12th Dhul-Hijjah. All of Mina is covered with Teflon coated tents to provide temporary accommodation to the pilgrims, thus termed as the world's biggest tent city. There are around 6,000 tents in approximately $20 \mathrm{~km}$-covered area of Mina. The salient aspects of tent city include:

- Each main group comprises 5000 persons and is allocated 250 tents, bringing density to 20 persons per tent ( $0.8 \mathrm{~m} 2$ average space per person). 250 tents each $4 \mathrm{~m} \times 4 \mathrm{~m}$ would cover $4000 \mathrm{~m} 2$. The layout of tents can be adjusted to site conditions, $80 \mathrm{mx} 50 \mathrm{~m}$ or any other configuration. The main tent groups are linked with walkways of around $2 \mathrm{~m}$ wide.

- Mina is a narrow valley served by a road network with primary orientation towards Muzdalifah / Arafat / Haram, especially major roads and cross links at the ground level. Whereas, elevated 6-lanes Highways cross Mina in East-West direction linking Azizyah / Haram.

- The road network serving many such groups consist of local, collectors and arterial roads.

- Typically, the local roads are undivided two-lane roads $(3.65 \mathrm{~m}$ each lane with 1 to $2 \mathrm{~m}$ shoulders on both sides) usable width for pedestrians can be taken as $9 \mathrm{~m}$. Collectors, generally 4-lanes divided highways (two lanes in each direction) total width around 20 $\mathrm{m}$, usable width $9 \mathrm{~m}$ in each direction. Arterials, generally 6-lanes divided highways (3 lanes in each direction of $3.65 \mathrm{~m}$ ), 27-30 m total width, with $12 \mathrm{~m}$ usable width on either side. The ramps are generally of $9 \mathrm{~m}$ width. The pedestrian walkways / blvds are of varying dimensions. The main pedestrian blvd in Mina can be taken as $20 \mathrm{~m}$ wide.

- With pedestrian density approaching to critical, the flow can be taken as 1 persons / m.s, with speed of 1 meter / second and density of 1.6 person / m2.

- Mina - Muzdalifah - Arafat are also linked with 18-km Metro, served by well dispersed 9 railway stations 3-each in Mina, Muzdalifah and Arafat. Seventeen 12-car trains, each capable of accommodating 3000 passengers move in convey of three at 90-second headways with peak design capacity of 72000 passengers per hour per direction.

- Jamarat complex is a ground plus 4-level structure, served by ramps connecting each level with one-way flow, a great improvement over the past practices [Rog 02]. Movement toward Azizyah / Haram is allowed, however cross movements are restricted. Metro station located next to Jamarat is connected to 4th level. Various levels are also linked with stairs and electric escalators for any emergency movements. Out of four days at Mina, Jamarat can be used for relief activities one day only (i.e, 8th Dhul-Hijjah) as a staging place for victims, whereas, for remaining three days (i.e, 
10-12 Dhul-Hijjah) it will be heavily crowded and can only serve as a transit point for evacuation by Metro/bus/helicopter/on foot to Azizyah/Haram.

- Notwithstanding the significance of layout and capacity of existing road network, the operational management of road network is of prime importance since it is synchronized with ongoing Hajj activities and is highly variable to accommodate prevailing environmental constraints, even allowing contra-flows.

- Hajj activities envisage that at the time of pilgrims' stay at Mina, Muzdalifah and Arafat will be available for accommodating any victims. However, in case of floods, the layout of valleys and historic flood flow pattern indicate that Arafat will be even more vulnerable than Mina since located near the catchment of 'Wadi Uranah'. Besides, keeping in view the mandatory Hajj rituals, pilgrims will prefer to stay close to Haram, whereas Arafat is even outside the Haram boundaries.

- Mina is well served by potential relief sites comprehensively linked with road network and essential services are also currently available, which are gradually shrinking in the wake of ongoing accelerated pace of urbanization / sprawl. 


\section{F Framework for Disaster Relief Plan}

Basic Considerations: The selection of basic parameters were done with utmost prudence due to its significant impact on efficiency of operational management [Klh 07, Zah 06]. Consequently, it is assumed that envisaged plan should:

- Address all potential disaster scenarios, like floods, fire, terrorist acts, stampede, etc, with focus on floods which could cause wide spread displacement.

- Be compatible with temporal dimensions of Hajj activities.

- Be simple, flexible and based on existing infrastructure and services.

- Optimize current resource management, while suggesting mechanism for further capacity building.

Outline Plan and Evacuation Sites: Following are salients of evacuation plan:

- Evacuation plan based on: multimodal transportation, on foot, by road and by Metro.

- Five evacuation areas have been designated adjoining Mashaaer e Mugadassah or areas between Holy places and Haram.

- Potential relief sites mainly encompassing: (1) Muzadalifah; (2) on Rd Makkah - Al Haddah near Faisal Bridge (4th ring road) Parking; (3) on King Abdul Aziz Rd near Mahbusul Jinn Parking, (4) on 3rd Ring Road near Kudai including areas east of Kudai - Ajyad tunnel; and (5) Rd As Sail Road near Al Sharai (marked on the map).

- Whereas, proposed evacuation site (1) is served with well laid out infrastructure for move on foot, road or Metro, alongwith well developed services to readily accommodate disaster victims, whereas the sites (2) - (5) have to be developed alongwith essential services and tentage with warehousing facilities.

- The multimodal evacuation plan encompasses 1-2 km self-evacuation (on-foot), 3-5 km by road transport and $6-12 \mathrm{~km}$ by Metro. The self-evacuation will be to the designated pick up points, i.e, Jamarat complex, Metro stations, existing bus stops, or temporarily established emergency pick up points, which remains the first phase of this research. 


\section{G Way Forward.}

- The study being of strategic dimensions made valuable recommendations to effect improvement in broad spectrum of activities pertaining to Hajj and Umrah. The salient being:

- Evolve long-term disaster risk management strategy and deploy modern decisionmaking tools for comprehensive planning and development.

- Deploy necessary hardware to develop an intelligent corridor in Mina to test the efficacy of tool prior to its induction for operational management of Hajj and Umrah activities.

- Undertake surveys for digitized mapping / modeling, arrange monitoring of environment and deploy GIS infrastructure for technology-smart management of mega events [Kos 06].

- Undertake floods risk assessment, institute structural / non-measures for risk mitigation, deploy effective warning system and ensure contingency planning based on sound flood management strategy for timely response [Daw 11].

- Undertake capacity building of institutions and develop human resource to measure up to the challenge.

- Focus on sustainable and integrated development of transportation system in Makkah for disaster risk reduction being in harmony with environment, economy and social aspects [Ras 05].

- Develop designated areas to be converted into proper relief camps at short notice to handle emergent situations and launch awareness campaign to educate the masses.

- Launch next phase of the research to extend calibration of the tool across entire Mashaaer Al Mugaddassah and Masjid Al Haram. 